\title{
A fast, stable dissemination protocol based on similarity of congestion level in urban vehicular networks
}

\author{
Haitao Xua ${ }^{\text {a }}$ Zhibei Qi \\ Urban Computing and Big Data Laboratory, Hangzhou Dianzi University, Hangzhou, Zhejiang, \\ China \\ axuhaitao@hdu.edu.cn, ${ }^{\mathrm{b}}$ qizhibei2010@163.com
}

Keywords: VANET, Vehicle density, Congestion similarity, Link quality, Forwarding node

\begin{abstract}
Aiming at the phenomenon that the current traffic congestion detection scheme has a long cycle and low efficiency of the information transmission in the city, this paper proposes a traffic congestion detection and congestion information forwarding scheme based on Vehicular Ad-hoc NETwork (VANET) technology. Based on vehicle-to-vehicle communication mode, the scheme first determine the occurrence of traffic congestion, through the congestion similarity theory combined with the communication link quality factors, select the appropriate congestion information forwarding nodes, achieve real-time rapid dissemination of information, reduce the amount of redundant information congestion purposes. Finally, the simulation results show that the proposed scheme has significant advantages in terms of information loss rate, propagation delay and forwarding rate and effectively improve the efficiency of traffic congestion information dissemination.
\end{abstract}

\section{Introduction}

Now more and more vehicles led to severe urban traffic gridlock, which seriously affected the development of the city. In order to improve urban traffic congestion, monitoring and detection technology emerges. When congestion occurs, it is important to rapidly determine the congestion areas, timely transmit of information and take appropriate measures as soon as possible.

The traditional congestion detection methods using a number of techniques to collect traffic information such as roadside magnetic loop detectors, surveillance cameras, wireless vehicle sensors, pressure pads, roadside radar and infra-red counters. These centralized approaches could successfully detect traffic jams, but if the testing equipment damage, some areas could not be monitored. A novel congestion detection technology was coming into view, which is Vehicular Ad-hoc Network (VANET). This technology completes the collection and exchange of information through the wireless communication between vehicles. VANET technology [2] derived from Mobile Ad-hoc Network (MANET), it mainly rely on wireless communication network. VANET is the distributed congestion detection scheme and each vehicle could become the center of network, 
and could be applied not only to detect congestion, but also has great application prospects in congestion information broadcast.

This paper uses the VANET technology and similarity of congestion level, proposed a fast and real-time congestion detection and information transmission scheme. The scheme drew on the trigger detection scheme for traffic congestion and flooding node forwarding optimization programs to reduce broadcast storm.

The remainder of this paper is organized as follows. In Section 2 we give an overview of the most significant related works. Afterwards, we propose the congestion detection. In Section 4, mainly explain the congestion information forwarding nodes selection. The simulation results we present in Section 5. Finally, the conclusion and future work are outlined in Section 6.

\section{Related Work}

It is very important that congestion information was collected by VANET. The paper [3] proposes a typical congestion detection method. A neighbor table would be established in each vehicle, recording the vehicles and roads information. The congestion could be quickly detected through the information of neighbor table and congestion detection standards. In this method, each vehicle could become an information network server (INS), but too many communications resources will be wasted vehicle information exchange. We have adopted the triggered detection scheme that the vehicle would start the communication process when congestion occurred. Under normal traffic conditions, vehicle traffic is not too often, the occurrence of a large amount of waste of communication resources can be avoided.

The dissemination of information related congestion is a multi-hop process in the congested area (or road). The appropriate approaches of "jump" need to be selected in order to get the information disseminated from the end to the other end. In paper [4] was introduced to select the intermediate forwarding nodes using multi-hop connectivity. In [5], the link quality was considered, in order to ensure the success rate of information-based intermediate node selection. The Greedy Perimeter Stateless Routing (GPSR) [6] protocols adopt the "greedy forwarding" scheme, the method selects the nodes that are closer to the destination node or area as forwarders. GPSR was the routing protocol based on the location, its advantage was that the routing protocol is relatively simple, the time delay of information transmission is short, and what was more, there was a good connectivity between nodes. But the shortcomings of GPSR were obvious, for example, the intermediate nodes too much, the overall delay too long and so on. Contention-Based Forwarding (CBF) [7] was also a kind of routing protocols based on the location. The difference was that the receivers were through "competition" to decide who would be the next forwarding node. This method could avoid that the source node information would be forwarded by too much nodes. The competitive mechanism could improve the performance of information forwarding scheme, for example, if the GPSR join the competition mechanism, which will reduce the forwarding node to a large extent. The timer is one of more commonly used in a competition mechanism [8], according to the routing protocols, the different receiving nodes would be given different forwarding waiting time, the shorter the waiting time, the higher the forwarding priority.

In fact, forwarding node evaluation criteria, there are two main aspects: number of intermediate nodes and communication quality. The number of intermediate nodes has a considerable impact on forwarding delay. If the distance is far, the number of intermediate nodes will be relatively small from one end to the other end. The communication quality is related to the success rate of information dissemination and communication efficiency, link quality is usually used as an indicator of communication quality. The higher the link quality, the higher the success rate and efficiency of information dissemination. In the paper [9] introduced the mechanism of such a choice 
of forwarding nodes, when the distance factor and link quality factors be considered at the same time, the information dissemination would have very high information transmission rate of success.

The node forwarding scheme in this paper is based on congestion detection, using vehicle density to obtain the similarity of congestion, and to determine an optimal forwarding distance, then we will select a node the most close to this position as a forwarding node, and take the source node congestion information forwarded to other cars around. At the same time, the introduction of competition mechanism and communication quality factors, which reduces communication time between the vehicles, information quantity and the burden on the communication in the premise of ensuring the success rate of communication. In addition, we will also discuss the congestion information dissemination scope and early warning of regional problems.

\section{Congestion detection scheme}

To begin with we need to determine the occurrence of congested roads or regions. Common method is to collect the environment information and itself information through own vehicle, and the changes of such information could judge whether the vehicle is in the congestion area or the area is congested roads. The indicators using to detect congestion mainly include vehicle speed change, vehicle density, travel direction, the unit delay and link saturation [10]. The characteristics of the congested roads are generally low speed and high density, so the speed and the density of vehicles is the two important indicators. This paper would mainly rely on the change of vehicle speed, the surrounding vehicle density, the direction of vehicle travel and the location of the vehicle to determine the congestion occurs, these four factors can quickly determine whether the vehicle is in the state of congestion. The definition of congestion is as follows:

$$
\mathrm{F}=(\mathrm{S}, \mathrm{D}, \mathrm{P}, \mathrm{Dir}) \text {. }
$$

Where $F$ is the state of congestion, $S$ is the changes of the vehicle speed, $D$ is regional vehicle density, $P$ is the vehicle position and Dir is the direction of travel.

Vehicle density refers to the number of vehicles in a region, many articles have introduced traffic density estimation scheme, such as the method of using street number and intersection number SJ ratio to estimate traffic density $[11,12]$.

We divide the vehicle speed into three levels: A, B, C. A is the normal speed of the vehicle, C is the speed is very small, and B is a critical speed, the relationship of the speed: $A>B>C$. We assume that the speed of the vehicles in the congested area less than $B$, the speed of the vehicles outside the congested area higher than B. Then the conversion process of vehicles speeds from A through B to reach $\mathrm{C}$, which can be regarded as one of the indicators of congestion (not the only standard). For the vehicle density, when the vehicle speed is less than B, the greater the density of the vehicle, the greater the possibility of congestion will be.

Definition 1 (congestion front-end): In the urban road, if the speed of the vehicle V less than B (namely $\mathrm{C}$ ) and vehicle density $\mathrm{D}$ around in a critical value within a certain period of time, when the vehicle speed from $\mathrm{C}$ to $\mathrm{B}$, and continue to rise trend to greater than or equal to $\mathrm{B}$, then we can identify $\mathrm{V}$ in front of the congestion, it can represent congestion region of the front-end critical position.

Such as shown in Fig. 1, if vehicle information transmission radius is $\mathrm{R}$, the vehicles are in $\mathrm{R}$, vehicle density $\mathrm{D}$ greater than critical density values, the $\mathrm{V} 1$ speed $\mathrm{S}$ in $\mathrm{C}$, and then $\mathrm{S}$ began to a promotion, passes through the critical value of $\mathrm{B}$. we can determine the vehicle V1 just out of the congested area, and congestion occurs at its rear area. V1 will generate congestion information which record vehicles position, velocity, orientation, density and Congestion judgment result, and 
send it to surrounding vehicles, the direction of information propagation is opposite direction of car traveling.

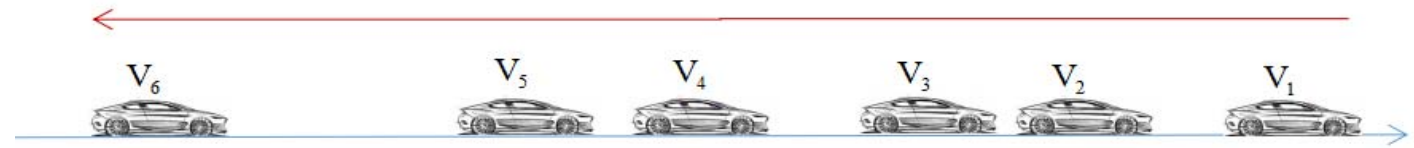

Fig. 1. Vehicle travel direction (blue line) and the direction of information dissemination (red line)

As previously defined of the congestion front, we also need to define the end of the congestion in order to determine the congested roads or congestion areas and congestion length. The end of the definition is similar to the definition of the front end.

Definition 2 (congestion rear-end): within a certain period of time, the vehicle speed in a normal state of speed, then slows down to below the critical speed B., vehicle density in the critical density values. At the same time, the vehicles received the congestion information from the front vehicle, then we can determine the vehicle belongs to the end position of the traffic congestion, which we call the end of the congestion.

When congestion occurs, the congestion front-end as the source node congestion information, sent information to the surrounding vehicles, through intermediate nodes constantly forwarding, the information arrived vehicle at the end of the congestion. The congestion rear-end is a processing terminal, it will analysis that road congestion intensity and length.

\section{Next forwarders selection}

After determining the congestion, the appropriate scheme of intermediate forwarding nodes selection can shorten the information propagation delay.

The congestion similarity. Generally speaking, vehicle can get their own vehicle density that according to the number of nodes around the vehicle within a certain range. Such as within the communication range, if the vehicle density is large, the vehicles state, environment information, the information received and the congestion strength are more similar. So we call congestion similarity of these vehicles is relatively high. Obviously, for the vehicles of high congestion similarity, their information is similar, if these vehicles are selected forwarding node, which is bound to create a lot of repeated or similar information redundancy. Based on the above theory, we can make such a conclusion: if the source node density is relatively large, the nodes around will have relatively high congestion similarity, at this time we should select the forwarding node as far as possible away from the source node, and vice versa.

Based on the above conclusions, this paper proposes a method of selecting forwarding nodes. This method essentially based on the position, which determined the optimal distance from source node to next forwarding node, and ensure the success rate of information transmission. The nodes of the most close to the optimal distance will be selected as the forwarding nodes, meanwhile we would adopt competitive mechanism to avoid forwarding flooding. For determining the size of the congestion similarity, we must first know the node density of the source node. According to the number of nodes (range of once hop) around the source node, we could determine the node density. Thus, we could determine the level of congestion similarity: the greater the node density, the higher the congestion similarity, the farther the distance between the forwarding node and the source node. The density of each node is different, and the distance from the source node to the next forwarding node is also different. The definition of node density is as follows: 
$\mathrm{D}_{\text {density }}=$ (Position, num node $_{\text {, }}$ Direction, Lane).

Position indicates the position of the current node, Num node represents the number of nodes around the current node, Direction is the direction of the node's movement and Lane is the number of lanes.

It is able to determine the level of congestion similarity according to the node density of the source node, and calculate the distance between the source node and the forwarding node, which we call the optimal distance. In once hop communication range, the optimal transmission distance which is calculated according to the density of nodes is less than information propagation distance ideally, so the method can improve the success rate of information dissemination with the effect of the environment and attenuation of signal. The formula for calculating the congestion similarity and the optimal distance is as follows:

$$
\begin{aligned}
& \mathrm{C}=\left(\mathrm{D}_{\text {density }} / \mathrm{M}\right) \beta . \\
& \mathrm{D}=\mathrm{S}-\alpha \mathrm{C} .
\end{aligned}
$$

$C$ is the congestion similarity, $D$ is the optimal distance, $S$ is the distance of information single hop in the ideal case, $M$ is the maximum number of vehicles within the range $S, \alpha$ and $\beta$ is adjust parameters.

Node received information from the source node, and which obtain a forwarding probability value according to the distance between two nodes and the optimal distance position, The closer to the optimal distance position, the larger the value of the forwarding probability, the higher the probability that the node becomes the forwarding node. When a node is located in the optimal range, the node's forwarding probability value gradually increase with the distance the farther away from the source node, if a node outside the optimal range, its forwarding probability decreases as the distance the farther away from the source node. The forwarding probability value formula is obtained according to the optimal distance:

$$
\begin{aligned}
& \mathrm{D}_{\mathrm{p}}=\left(\mathrm{D}_{\text {node }} / \mathrm{D}\right) \gamma \\
& \mathrm{D}_{\mathrm{p}}=\left(1-\left(\left(\mathrm{D}_{\text {node }}-\mathrm{D}\right) / \mathrm{D}\right)\right) \gamma \quad \text { when } \quad \mathrm{D}_{\text {node }}<\mathrm{D} . \\
& \text { when } \mathrm{D}_{\text {node }}>\mathrm{D} .
\end{aligned}
$$

$D_{\text {node }}$ is the actual distance between the receiving node, the source node. $\gamma$ is distance weight, and the $\mathrm{D}$ is the optimal distance.

The link quality factors. The original purpose of reducing intermediate forwarding node is to reduce the forwarding time and information redundancy, but this method cannot guarantee success rate of information dissemination. In fact, due to external interference, signal attenuation and so on, it is very difficult to ensure the dissemination success rate with a minimum of intermediate nodes. The success rate of forwarding information must be considered, we get a forwarding probability when calculate the optimal distance finally, that is, the closer to the optimal distance position, the higher the probability that the candidate node becomes a forwarding node. Considering link quality (communications quality), it is also provides a probability that the candidate node becomes a forwarding node actually, the better the link quality, the higher the success rate of information dissemination. So we need to do is that synthesizing these probability factors, and choosing one of the best forwarding nodes based on these aspects. The formula for defining a node's forwarding probability can be defined:

$$
\mathrm{P}=\omega_{\mathrm{p}} \mathrm{Q}+\left(1-\omega_{\mathrm{p}}\right) \mathrm{D}_{\mathrm{p}}
$$

$Q$ is the link quality factor (a probability value), $D_{p}$ is the distance probability, $\omega_{p}$ is the parameter between $0 \sim 1$.

The link quality factors contains many aspects, such as communication signal and the channel quality, transmit power, environmental noise and so on, We mainly use the information 
dissemination success rate and propagation delay to detect link quality. Firstly, the strength of the received signal, which will have varying degrees of change with distance changes, we will abandon the weak signal, and considering only the strong signal. $R S_{t h}$ is the critical value of , $R S_{\max }$ is the maximum strength of received signal, Only signals of the strength between $R S_{t h}$ and $R S_{\max }$ will be considered, namely received signal strength $R S \in\left(R S_{t h}, R S_{\max }\right)$.

Then the noise factors, SNR is the signal-to-noise ratio, which is the ratio of signal power and noise power.

$\mathrm{SNR}=$ Psignal $/$ Pnoise

$P L R$ is the packet loss rate, which could represent information transmission success rate:

PLR $=$ Packetlose $/$ Packetstotal.

With the above theory, we can get the formula of link quality:

$$
\mathrm{Q}=\mu \mathrm{RS}(1-(1 / \mathrm{SNR}))+(1-\mu)(1-\mathrm{PLR}) .
$$

$Q$ indicates the link quality that is a probability value, $\mu$ is a link quality weight between 0 and 1 . According to the forwarding probability values, each node will set different forwarding waiting time. The waiting time of nodes having maximum forwarding probability will be relatively short, thus this node have the highest priority for transmitting, when the forwarding node forwarding information, the nodes received information from the source node, again receive information from the forwarding node, then which will abandon the information of the source node. The formula for forwarding the waiting time as follows:

$$
\mathrm{WT}=\mathrm{T}(1-\mathrm{P})+\delta \text {. }
$$

$T$ is the cycle of information transmission, which is the time to send a message queue. $P$ is the forwarding probability. $\delta$ is a random number less than T, and its purpose is to prevent the nodes of the same probability to forwarding information at the same time.

\section{Performance evaluation}

In this section, we aim to do the relevant simulation experiments, such as the simulation of urban road by JOSM, simulation of the vehicle node in the mobile process by SUMO, and simulation of the process of forwarding information by NS-2. We would compare the optimal distance scheme and the common in two ways: greedy forwarding and the most distance forwarding scheme.

Simulation setting. To prove the efficiency of our forwarding mechanism, we need to simulate traffic state. Using JOSM and SUMO simulation software, we can simulate the urban road topology and the driving conditions of vehicle, finally we could complete the collection of relevant data. Next, the NS-2 simulation experiments could complete collection of the communication data.

In particular, we implemented and tested these schemes simulator with IEEE802.11p, and selected the most realistic and representative models for vehicular communications. The complete list of simulation parameters is given in Table 1. 
Table 1 Simulation parameters.

\begin{tabular}{|l|l|}
\hline Frequency band & $5.5 \mathrm{GHz}$ \\
\hline Bandwidth & $10 \mathrm{MHz}$ \\
\hline Transmission range & $200 \mathrm{~m}$ \\
\hline Propagation delay & $20 \mathrm{us}$ \\
\hline Data message size & $100 \mathrm{bytes}$ \\
\hline $\mathrm{RS}_{\text {th }}$ & $-40 \mathrm{dBm}$ \\
\hline$\alpha, \beta$ (adjust parameters) & 1.5 \\
\hline$\mu$ (link quality weight) & 0.6 \\
\hline$\omega_{\mathrm{p}}$ (forwarding probability parameters) & 0.5 \\
\hline Speed of vehicle & $0 \sim 100 \mathrm{~km} / \mathrm{h}$ \\
\hline$\delta$ (random number) & $1 \sim 10 \mathrm{us}$ \\
\hline$T$ (transmission cycle) & $20 \mathrm{us}$ \\
\hline$\gamma$ (distance weight) & 0.5 \\
\hline Broadcast frequency & $1 \mathrm{~Hz}$ \\
\hline Noise level & $-100 \mathrm{dBm}$ \\
\hline
\end{tabular}

Forwarding mechanism evaluation. To prove the efficiency of the optimal distance forwarding mechanism against the other schemes, we set our protocol according to different evaluation index. The evaluation metrics considered are (i) average packet loss rate, (ii) the total delay.

As can be seen from Fig. 2, the packet loss rate of the three node forwarding schemes is different in different vehicle density. The most distant option scheme, its packet loss rate did not particularly obvious changes with change of vehicle density, the main reason is that the most distance forwarding scheme is only related to the communication range, and not affected by the density of the vehicle. Although the relationship between greedy forwarding scheme and vehicles density is not closely related, but due to the increased density, which makes the increase in intermediate forwarding nodes, and multiple forwarding nodes also would forwarded similar information, with the density increasing and information transmission distance longer, the packet loss rate of the greedy forwarding scheme will gradually rise, without a doubt, it has maximum number of packet loss.

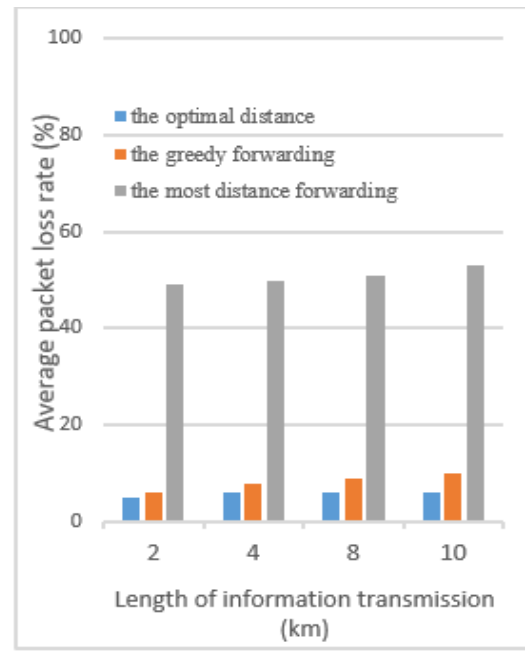

A、 $100 \mathrm{veh} / \mathrm{km} 2$

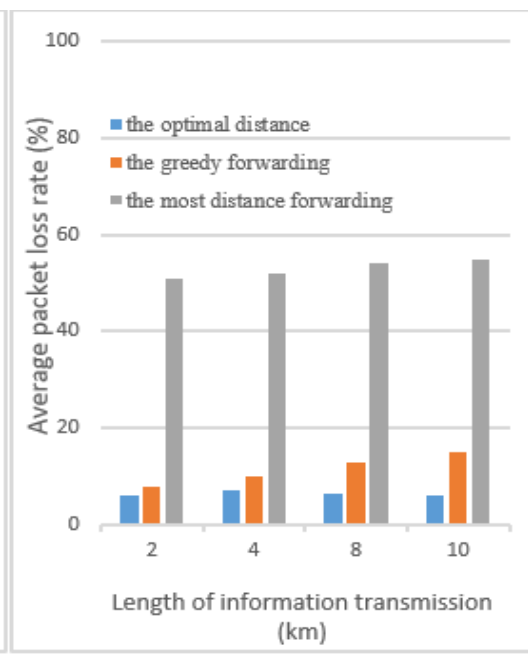

A、 $200 \mathrm{veh} / \mathrm{km} 2$

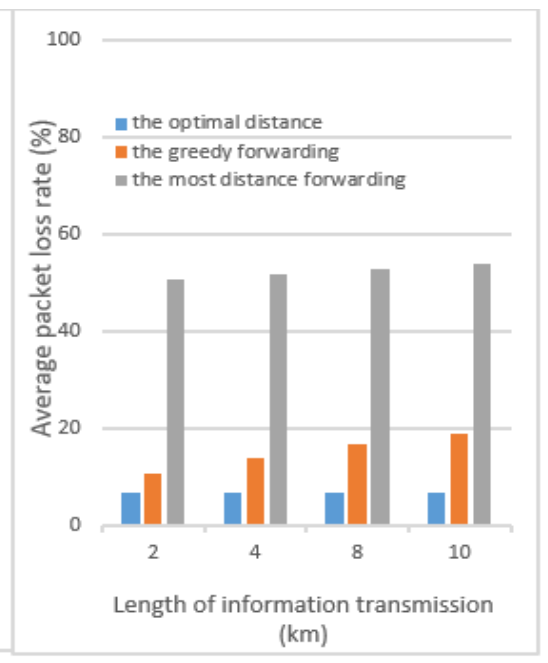

A、 $300 \mathrm{veh} / \mathrm{km} 2$

Fig. 2. Average packet loss rate under different vehicle density and length of information transmission 
As shown in Fig 3, three kinds of information transmission scheme based on the change of the vehicle density and transmission distance, their total delay will gradually increase. For the most distant forwarding scheme, its forwarding distance is relatively stable each time, so the delay rise would be more stable, that is, the delay increment of this scheme is a constant. To the greedy forwarding, with the increase of density, the number of forwarding node will gradually become more, thus the processing delay and transmission delay will increase significantly.

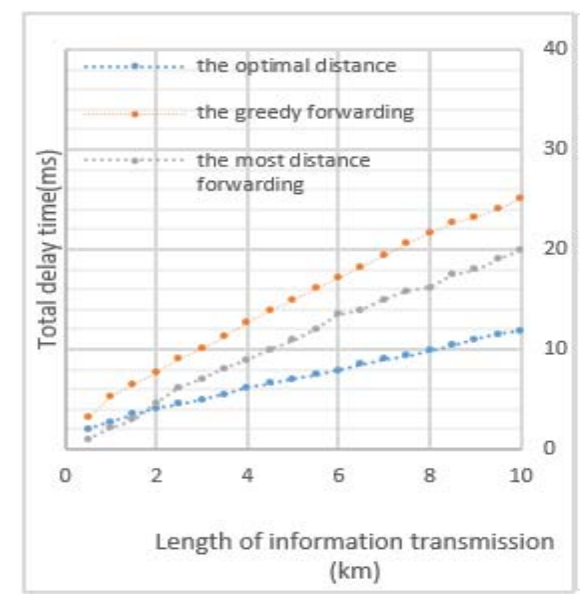

A 、 $100 \mathrm{veh} / \mathrm{km} 2$

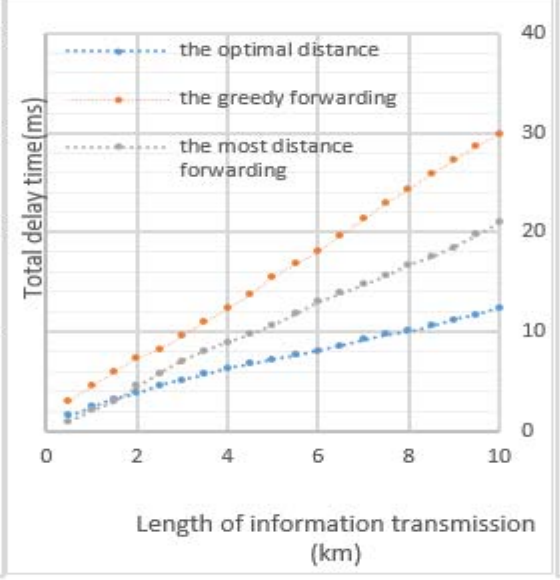

A、 $200 \mathrm{veh} / \mathrm{km} 2$

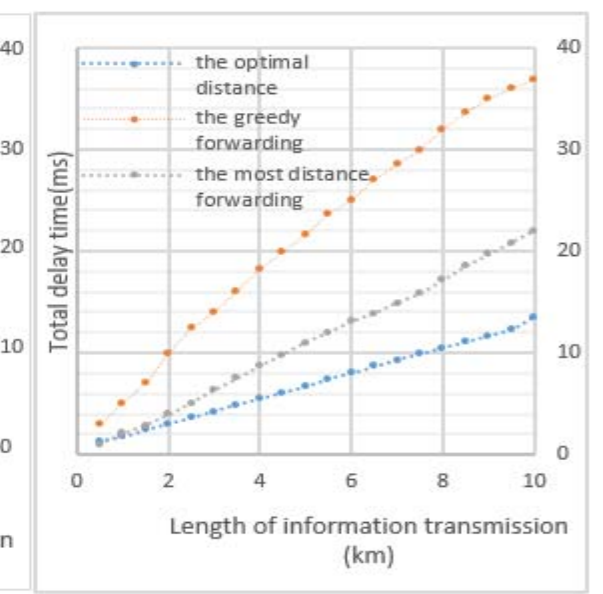

A $300 \mathrm{veh} / \mathrm{km} 2$

Fig. 3. Total delay time under different vehicle density and length of information transmission

We can see from the curve of Fig 6, the optimal distance forwarding scheme with the increase of the density of the vehicle, its delay hardly increase. We take advantage of the 20 different transmission distance to draw the curve diagram in the graph, when the vehicle density is 200 , and have no regard of irregular fluctuation what is caused because of the random distribution of vehicles, there was no obvious change in the delay of optimal distance forwarding, namely, the greater the density of vehicles, the less obvious the delay increases. The reason is that the optimal forwarding distance would be gradually increased with the increase of the vehicles density, resulting in the scheme would tend to the most remote forwarding scheme, but due to limitations of link quality factors, the optimal forwarding distance increases would more and more slowly with the increase of density, ideally, when the vehicle density reached a certain degree, the optimal distance will stabilize at a value less than the propagation distance.

From the viewpoint of evaluation results of experiments, the advantage of the optimal distance forwarding scheme is that could quickly spread traffic congestion information, small delay, high stability, low packet loss rate, and forwarding nodes are relatively less because of the existence of competition mechanism, therefore, it can very efficient transmission congestion information.

\section{Conclusions}

The paper proposed a congestion information forwarding scheme optimal forwarding distance based on the VANET technology. The scheme solve the traffic congestion detection and information transmission, to achieve the optimization of congestion information transmission method. On the other hand, application of VANET technology in terms of traffic is far from fully developed, and with researchers more and more carries on the thorough excavation, we believe that the future of VANET technology in the field of traffic more glory. 


\section{Acknowledgements}

This work was financially supported by Public Projects of Zhejiang Province (2013C33082, 2015C33067), State Key Program of Zhejiang Province Natural Science Foundation of china (LZ15F020003) and Zhejiang Provincial Natural Science Foundation of China (LY12F02017).

\section{References}

[1] Darwish T, Bakar K A. Traffic density estimation in vehicular ad hoc networks: A review[J]. Ad Hoc Networks, 2014, 24:337-351.

[2] Hyun Yu, Joon Yoo, Sanghyun Ahn. A VANET Routing based on the Real-time Road Vehicle Density in the City Environment[J].IEEE(2013)978-1-4673-5990-0/13

[3] Quan Yuan, Zhihan Liu,Jinglin Li. A traffic congestion detection and information dissemination scheme for urban expressways using vehicular networks.Transportation Research Part C 47 (2014) 114-127

[4] M. Rondinone, J. Gozalvez. Distributed and Real Time Communications Road Connectivity Discovery through Vehicular Ad-hoc Networks[C].2010 13th International IEEE Annual Conference on Intelligent Transportation Systems Madeira Island,Portugal,September(2010)19-22

[5] Chi Trung Ngo, Hoon Oh. A Link Quality Prediction Metric for Location based Routing Protocols under Shadowing and Fading Effects in Vehicular Ad Hoc Networks[J]. Procedia Computer Science 34 ( 2014 ) $565-570$

[6] B. Karp and H. T. Kung. GPSR: greedy perimeter stateless routing for wireless networks[J]. in Proc. ACM/IEEE MOB[COM'OO, Boston,Massachusetts, USA, 2000, pp.243-2S4

[7] H.FussIer,1.Widmer,M.Kasemann,M.Mauve,H.Hartenstein.Contention-based forwarding for mobile ad hoc networks[J]. in Ad-Hoc Networks, vol.I, issue 4, pp.3SI-369, Nov. 2003.

[8] Ramon Bauza,Javier Gozalvez,Joaquin Sanchez-Soriano.Road Traffic Congestion Detection through Cooperative Vehicle-to-Vehicle Communications[J]. IEEE(2010) 978-1-4244-8389-1/10

[9] Sofiane Zemouri, Soufiene Djahel, John Murphy. A fast, reliable and lightweight distributed dissemination protocol for safety messages in Urban Vehicular Networks[J].Ad Hoc Networks 27 (2015) 26-43

[10]JIA Hongfei,TAN Yunlong,Fu Xiaoyan. Research and Application of Urban Traffic Congestion Evaluation System[J].2010 International Conference on Optoelectronics and Image Processing,IEEE(2010)978-0-7695-4252-2/10

[11][13]Javier Barrachina, Piedad Garrido, Manuel Fogue. I-VDE: A Novel Approach to Estimate Vehicular Density by Using Vehicular Networks[J].ADHOC-NOW (2013)LNCS 7960

[12] Julio A.Sanguesa, Manuel Fogue. An Infrastructureless Approach to Estimate Vehicular Density in Urban Environments[J].Sensors(2013)13,2399-2418 